\title{
Влияние свободных носителей заряда на двулучепреломление и дихроизм в слоях анизотропного пористого кремния
}

\author{
() К.С. Секербаев ${ }^{1,2}$, Е.Т. Таурбаев ${ }^{1,2}$, А.И. Ефимова ${ }^{3}$, В.Ю. Тимошенко ${ }^{3,4, \text {, Т.И. Таурбаев }}{ }^{1,2}$ \\ ${ }^{1}$ Научно-исследовательский институт экспериментальной и теоретической фризики \\ при Казахском национальном университете им. аль-Фараби, \\ 050040 Алматы, Республика Казахстан \\ ${ }^{2}$ Национальная нанотехнологическая лаборатория открытого типа \\ при Казахском национальном университете им. аль-Фараби, \\ 050040 Алматы, Республика Казахстан \\ ${ }^{3}$ Московский государственный университет им. М.В. Ломоносова (физический фракультет), \\ 119991 Москва, Россия \\ ${ }^{4}$ Национальный исследовательский ядерный университет „МИФИ“, \\ 115409 Москва, Россия \\ ๑ E-mail: vtimoshe@gmail.com
}

(Получена 13 декабря 2016 г. Принята к печати 25 января 2017 г.)

\begin{abstract}
Экспериментально и теоретически исследованы оптические свойства анизотропных пленок мезопористого кремния, содержащих свободные носители заряда (дырки), в инфракрасной области спектра. Результаты моделирования оптических свойств полученных образцов в рамках приближения эффективной среды демонстрируют сильную зависимость двулучепреломления, анизотропии отражения и дихроизма от концентрации свободных носителей заряда. Вкладом носителей заряда с концентрацией порядка $10^{19} \mathrm{~cm}^{-3}$ объясняются немонотонные зависимости спектров разностного пропускания образцов, измеренные при взаимно перпендикулярных направлениях поляризации света. Полученные результаты свидетельствуют о перспективности анизотропных кремниевых наноструктур для инфракрасной фотоники и терагерцовой техники.
\end{abstract}

DOI: $10.21883 /$ FTP.2017.08.44796.8119

\section{1. Введение}

Кремниевые наноструктуры, такие как слои пористого кремния (por-Si), полученные электрохимическим травлением кристаллического кремния (c-Si) в растворах плавиковой кислоты, проявляют уникальные электрические и оптические свойства, применимые в оптоэлектронике и фотонике [1-3]. В частности, кремний с анизотропной системой пор [4-6] обладает двулучепреломлением, которое может варьироваться в широком диапазоне при изменении пористости и диэлектрической проницаемости вещества, заполняющего поры [5,6]. Это может быть использовано, например, в нелинейной оптике для фазового согласования процесса генерации второй гармоники [2,6], а также в оптоэлектронике для создания устройств переключения света [7]. Благодаря развитой внутренней поверхности por-Si и чувствительности его электронных и оптических свойств к диэлектрической проницаемости окружающей среды данный материал может быть использован в газовых сенсорах, включая устройства с оптическим детектированием [7-9].

Несмотря на значительное количество работ по исследованию двулучепреломления и дихроизма в анизотропных образцах por-Si [2,4-6], детального исследования влияния концентрации носителей заряда на их оптическую анизотропию не проводилось. В настоящей работе представлены результаты экспериментального и теоретического исследования оптических свойств анизотропных слоев por-Si, выращенных на сильнолегирован- ных пластинах $c$-Si с различным уровнем легирования и ориентацией поверхности (110), которые указывают на сильную зависимость двулучепреломления и дихроизма в инфракрасной (ИК) области спектра от концентрации свободных носителей заряда.

\section{2. Методика эксперимента}

Образцы por-Si были получены стандартным методом электрохимического травления пластин $c$-Si проводимости $p$-типа (легирование бором) с сопротивлением $1-5,10-20$ и 30-50 мОм · см, имеющих оптическую полировку поверхности с ориентацией (110). В качестве электролита был использован водный раствор фтористоводородной кислоты, смешанный с этанолом (48\%) в соотношении $1: 1$. Процесс травления проводили при постоянной плотности тока $60-80 \mathrm{MA} / \mathrm{cm}^{2}$, что позволяло получать образцы с пористостью 50-70\%. Пленки por-Si были отделены от подложек кратковременным увеличением плотности тока до $700 \mathrm{MA} / \mathrm{cm}^{2}$. Как установлено в работе [4], используемый режим травления приводит к формированию так называемого мезопористого кремния с характерным размером кремниевых остатков (кристаллитов) и пор 10 нм и более, имеющих преимущественную ориентацию в плоскости пленки в кристаллографическом направлении [001]. Толщину пленки por-Si определяли с помощью оптического микроскопа Metam RV-22 (LOMO Ltd., Россия), она 
изменялась от 8 до 60 мкм с увеличением длительности электрохимического травления в интервале 5-40 мин.

Инфракрасные спектры пропускания и отражения отслоенных пленок por-Si были измерены с помощью Фурье-спектрометра Bruker IFS66v со спектральным разрешением $2 \mathrm{~cm}^{-1}$. Измерения проводили при нормальном падении поляризованного ИК излучения на образец при комнатной температуре на воздухе. При измерениях использовалось плоскополяризованное ИК излучение с направлением вектора электрического поля вдоль или перпендикулярно кристаллографическому направлению [001] в плоскости пластины исходного монокристалла $c$-Si. Исследовали свежеприготовленые пленки por-Si, что позволило анализировать ансамбли кремниевых нанокристаллов с максимально возможной концентрацией свободных носителей заряда, поскольку, как было установлено ранее, окисление поверхности por-Si приводит к уменьшению такой концентрации $[10,11]$.

\section{3. Моделирование оптических свойств}

В инфракрасном спектральном диапазоне пленку мезопористого por-Si можно рассматривать как однородную оптическую среду с эффективной диэлектрической проницаемостью, которую можно рассчитать по обобщенной формуле Бруггемана [2]:

$$
P \frac{\varepsilon_{\mathrm{eff}}-\varepsilon_{1}}{\varepsilon_{\mathrm{eff}}+L_{1}\left(\varepsilon_{1}-\varepsilon_{\mathrm{eff}}\right)}+(1-P) \frac{\varepsilon_{\mathrm{eff}}-\varepsilon_{2}}{\varepsilon_{\mathrm{eff}}+L_{2}\left(\varepsilon_{2}-\varepsilon_{\mathrm{eff}}\right)}=0
$$

где $\varepsilon_{\text {eff }}$ - эффективная диэлектрическая проницаемость, $P$ - пористость среды, $\varepsilon_{1}$ и $\varepsilon_{2}$ - диэлектрические проницаемости пор и нанокристаллов кремния соответственно, $L_{1}$ и $L_{2}$ - факторы деполяризации для пор и нанокристаллов кремния.

Для описания оптических свойств анизотропных пленок por-Si (110) обычно рассматривают ансамбли эллипсоидов вращения из нанокристаллов кремния и пор с равными факторами деполяризации $\left(L_{1}=L_{2}\right)$, зависящими от условий получения [2]. Поскольку размеры нанокристаллов в исследуемых мезопористых слоях имеют значения порядка 10 нм или больше, то при описании поведения носителей заряда в них квантово-размерные эффекты можно не учитывать, а оптические свойства в ИК диапазоне могут быть описаны классической моделью Друде-Лоренца [4,5]:

$$
\varepsilon_{2}=\varepsilon_{\infty}-\frac{\omega_{p}^{2}}{\omega^{2}+i \omega \tau^{-1}},
$$

где $\varepsilon_{\infty}-$ высокочастотная диэлектрическая проницаемость $\left(\varepsilon_{\infty}=11.7\right.$ для $c$-Si $), \omega_{p}-$ плазменная частота $\left(\omega_{p}=\sqrt{e^{2} N_{p} / m^{*} \varepsilon_{0}}\right.$, где $e-$ заряд электрона, $N_{p}$ - концентрация свободных носителей заряда (дырок), $m^{*}$ - эффективная масса носителей заряда, $\varepsilon_{0}=8.85 \cdot 10^{-12} \Phi / \mathrm{M}-$ диэлектрическая постоянная), $\tau$ - время релаксации квазиимпульса носителей заряда, $\omega$ - частота падающего излучения.

Анизотропия формы нанокристаллов кремния может быть описана фактором деполяризации, зависящим от отношения полуосей эллипса $x$, который в случае направления электрического поля вдоль оси вращения определяется выражением [1,5]:

$$
L_{\|}=\frac{1}{1-x^{2}}\left(1-x \frac{\arcsin \sqrt{1-x^{2}}}{\sqrt{1-x^{2}}}\right) .
$$

Для перпендикулярного направления поля соответствующий фактор деполяризации $L_{\perp}$ может быть определен из условия нормировки [5]: $L_{\|}+2 L_{\perp}=1$.

С помощью соотношений $(1-3)$ можно рассчитать эффективную диэлектрическую проницаемость por-Si и соответствующие оптические характеристики. Так, показатель преломления определяется из эффективной диэлектрической проницаемости следующим выражением:

$$
n_{o, e}=\overline{\frac{1}{2}\left(\operatorname{Re} \varepsilon_{\mathrm{eff}}^{\|, \perp}+\sqrt{\left(\operatorname{Re} \varepsilon_{\mathrm{eff}}^{\|, \perp}\right)^{2}+\left(\operatorname{Im} \varepsilon_{\mathrm{eff}}^{\|, \perp}\right)^{2}}\right)},
$$

где индексы $o$ и $e$ соответствуют обыкновенному и необыкновенному лучам, поляризованным соответственно перпендикулярно и параллельно кристаллографическому направлению [001] в плоскости пленки.

Коэффициент поглощения света для обыкновенного и необыкновенного лучей определяется стандартным выражением:

$$
\alpha_{o, e}=\frac{\omega I m_{\mathrm{eff}}^{\|, \perp}}{c n_{o, e}},
$$

где $c$ - скорость света в вакууме.

Коэффициенты отражения и пропускания (при нормальном падении) вычисляются из следующих соотношений:

$$
\begin{gathered}
R_{o, e}=\left(\frac{n-n_{o, e}}{n+n_{o, e}}\right)^{2}, \\
T_{o, e}=\left(1-R_{o, e}\right) e^{-\alpha_{o, e} d},
\end{gathered}
$$

где $n$ - показатель преломления граничащей среды, в данном случае границей является воздух $(n=1) ; d-$ толщина пленки por-Si.

Теоретический анализ вклада подвижных носителей заряда анализировали в диапазоне концентраций дырок $N_{p}$ от $10^{17}$ до $10^{20} \mathrm{~cm}^{-3}$. Дополнительными параметрами модели являлись две величины $x$ и $\tau$.

\section{4. Результаты и обсуждение}

На рис. 1 приведены спектры пропускания обыкновенного и необыкновенного лучей для достаточно толстой пленки por-Si, приготовленной из пластины $c$-Si с удельным сопротивлением $1-5 \mathrm{MOM} \cdot \mathrm{cm}$ $\left(N_{p}=10^{20}-2 \cdot 10^{19} \mathrm{~cm}^{-3}\right)$. Видно, что коэффициент пропускания для света, поляризованного вдоль кристаллографического направления [001] $T_{e}$ больше, чем для 


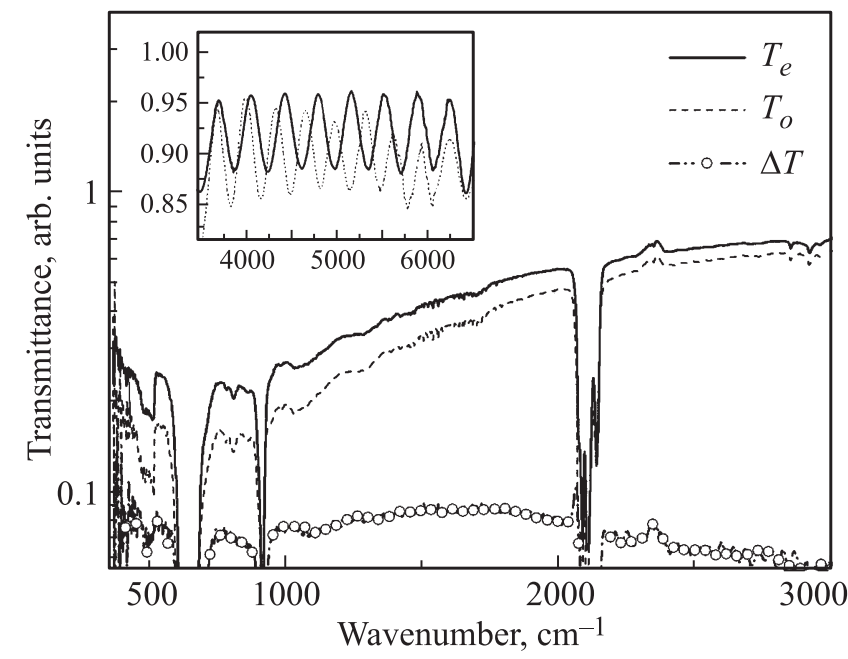

Рис. 1. Спектры пропускания анизотропной пленки por-Si $(d=60$ мкм, $P=60 \%)$, полученной из сильно легированных бором пластин $c$-Si с удельным сопротивлением $1-5 \mathrm{MOM} \cdot \mathrm{cm}$ и ориентацией поверхности (110), измеренные при поляризации света параллельно $\left(T_{e}\right)$ и перпендикулярно $\left(T_{o}\right)$ кристаллографическому направлению в плоскости пленки [001], а также их разности $\Delta T=T_{e}-T_{o}$. На вставке показаны спектры пропускания приготовленных в тех же условиях пленок por-Si толщиной 8 мкм.

перпендикулярной поляризации $T_{o}$. Данный факт объясняется анизотропией поглощения ИК излучения свободными носителями заряда [5]. Разность коэффициентов пропускания $\Delta T=T_{e}-T_{o}$ (кружки на рис. 1) проявляет слабо выраженную немонотонную зависимость от частоты ИК излучения, достигая максимальных значений в области $1500-2000 \mathrm{~cm}^{-1}$, что будет подробно обсуждено далее.

На вставке рис. 1 показаны фрагменты спектров пропускания пленки por-Si достаточно малой толщины, которые демонстрируют различный частотный период интерференционной модуляции пропускания для обыкновенного (пунктирная линия) и необыкновенного (сплошная линия) лучей. Это указывает на различие показателей преломления (двулучепреломление). Меньший период интерференционной модуляции для $T_{o}$ по сравнению с $T_{e}$, очевидно, свидетельствует о том, что изучаемые образцы представляют „отрицательный“ двулучепреломляющий кристалл $\left(n_{o}>n_{e}\right)$. Для образцов, приготовленных из подложек с удельным сопротивлением 1-5 мОм · см, показатели преломления в области слабого поглощения (5000-6500 $\left.\mathrm{cm}^{-1}\right)$ составили соответственно $n_{o}=2.0$ и $n_{e}=1.85$. Расчет по формуле (6) с использованием этих значений дает $R_{o}=11 \%$ и $R_{e}=9 \%$, что хорошо согласуется с результатами измерения спектров отражения и пропускания.

Из экспериментальных спектров пропускания были оценены величины коэффициентов поглощения $\alpha_{\text {exp }} \approx-\ln (T) / d$ для обыкновенного и необыкновенного лучей. Их разность (дихроизм) возрастает с уменьшени- ем волнового числа в согласии с расчетами в рамках используемой модели (рис. 2,a), в которой кремниевые нанокристаллы аппроксимированы эллипсоидами с соотношением полуосей $x=1.3$, концентрацией носителей заряда $N_{p}=2 \cdot 10^{19} \mathrm{~cm}^{-3}$ и величиной затухания $\tau^{-1}=500 \mathrm{~cm}^{-1}$. Величина $N_{p}$ близка к концентрации дырок в используемых подложках $c-\mathrm{Si}$.

Согласно данным моделирования, в отражении пористых пленок, приготовленных из подложек с удельным сопротивлением 1-5 мОм · см, имеется плазменный минимум вблизи $500 \mathrm{~cm}^{-1}$. В спектральной области выше $1000 \mathrm{~cm}^{-1}$ величины коэффициентов отражения составляют 8-10\%, их разность $\Delta R=R_{o}-R_{e}$ составляет примерно $1.5-2 \%$ и плавно возрастает с частотой в согласии с данными эксперимента (рис. 2,b). Следовательно, заметное $(\sim 10 \%)$ разностное пропускание $\Delta T$, показанное на рис. 1, обусловлено преимущественно анизотропией поглощения исследуемых пленок.

Расчет величины дихроизма $\Delta \alpha=\alpha_{o}-\alpha_{e}$ (рис. 3, $a$ ) и разности показателей преломления $\Delta n=n_{o}-n_{e}$ (рис. $3, b)$ свидетельствует об их различной зависимости от концентрации свободных носителей заряда. Точками на рис. 3 нанесены экспериментально измеренные значения для образцов, приготовленных на подложках с различной концентрацией носителей заряда. Видно, что наиболее близки к экспериментальным значениям расчеты при параметре анизотропии $x=1.3$. Разность
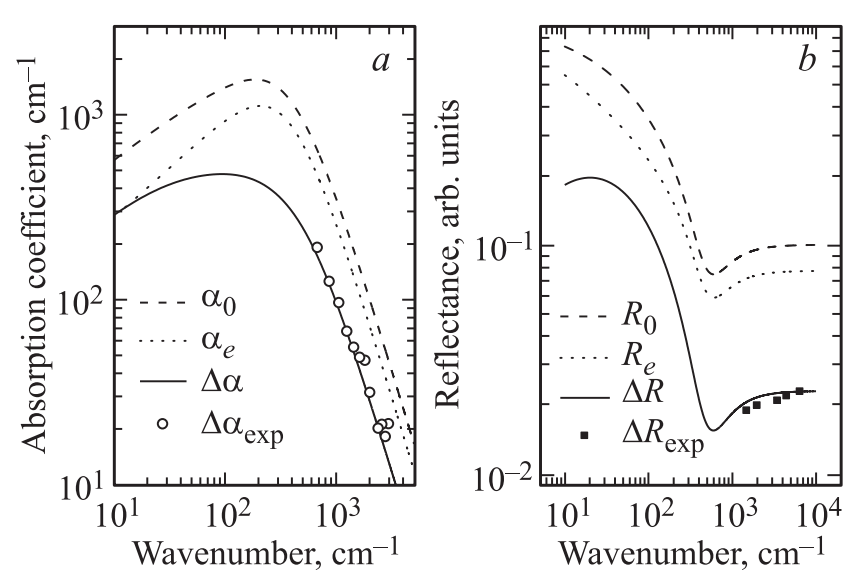

Рис. 2. $a-$ расчетные спектры коэффициента поглощения анизотропных пленок por-Si для поляризации света перпендикулярно ( $\alpha_{o}$, штриховая линия) и параллельно $\left(\alpha_{e}\right.$, пунктирная линия) кристаллографическому направлению [001], а также расчетные спектры дихроизма $\Delta \alpha$ (сплошная линия) и экспериментальные данные $\Delta \alpha_{\exp }$ (точки) для образца, полученного из сильно легированных бором $c$-Si пластин с удельным сопротивлением $1-5 \mathrm{MOM} \cdot$ см. $b-$ расчетные спектры отражения для поляризации света перпендикулярно $\left(R_{o}\right.$, штриховая линия) и параллельно $\left(R_{e}\right.$, пунктирная линия $)$ кристаллографическому направлению $[001]$, а также разность этих спектров $\Delta R=R_{o}-R_{e}$ (сплошная линия) и экспериментальные данные $\Delta R_{\text {exp }}$ (точки). При расчетах принималось: пористость $P=60 \%$, отношение полуосей $x=1.3$, величина затухания $\tau^{-1}=500 \mathrm{~cm}^{-1}$. 
показателей преломления слабо зависит от $N_{p}$ при низких концентрациях носителей, но при концентрациях более $10^{19} \mathrm{~cm}^{-3}$ величина $\Delta n$ монотонно уменьшается с ростом $N_{p}$ и даже меняет знак при $N_{p}>5 \cdot 10^{19} \mathrm{~cm}^{-3}$ (рис. $2, b$ ). В то же время дихроизм практически линейно нарастает с увеличением $N_{p}$ в анализируемом диапазоне концентраций (рис. 3, $a$ ).

Наблюдаемое различие зависимостей двулучепреломления и дихроизма от концентрации носителей заряда объясняется тем, что величины показателей преломления в равной степени зависят как от действительной, так и от мнимой частей компонент $\varepsilon_{\text {eff }}$ (формула (4)). При этом коэффициент поглощения света свободными носителями заряда пропорционален мнимой части $\varepsilon_{\mathrm{eff}}$, величина которой линейно зависит от концентрации носителей (формулы (2), (5)). При низких концентрациях носителей заряда, когда их вклад в $\varepsilon_{\text {eff }}$ незначителен, величина $\Delta n$ определяется только анизотропией действительных частей $\varepsilon_{\text {eff, }}$ что хорошо иллюстрируют кривые, рассчитанные для $x=1.2-1.5$ (рис. $3, b$ ). В то же время величины $\alpha_{o, e}$ при тех же концентрациях носителей практически пропорциональны мнимым частям соответствующих компонент $\varepsilon_{\text {eff, }}$ что приводит к линейной зависимости $\Delta \alpha$ от $N_{p}$ (рис. $2, a$ ).

Рис. 4 показывает расчетные спектры пропускания и разностного пропускания для пленок por-Si $(d=60$ мкм, $\left.N_{p}=2 \cdot 10^{19} \mathrm{~cm}^{-3}\right)$ в широкой спектральной области. Измеренный спектр $\Delta T_{\exp }$ близок к расчетному в диапазоне $1000-3000 \mathrm{~cm}^{-1}$, что указывает на обоснованность основных положений используемой модели, в частности, оценки концентрации носителей заряда и степени анизотропии пленок por-Si. Наблюдаемые
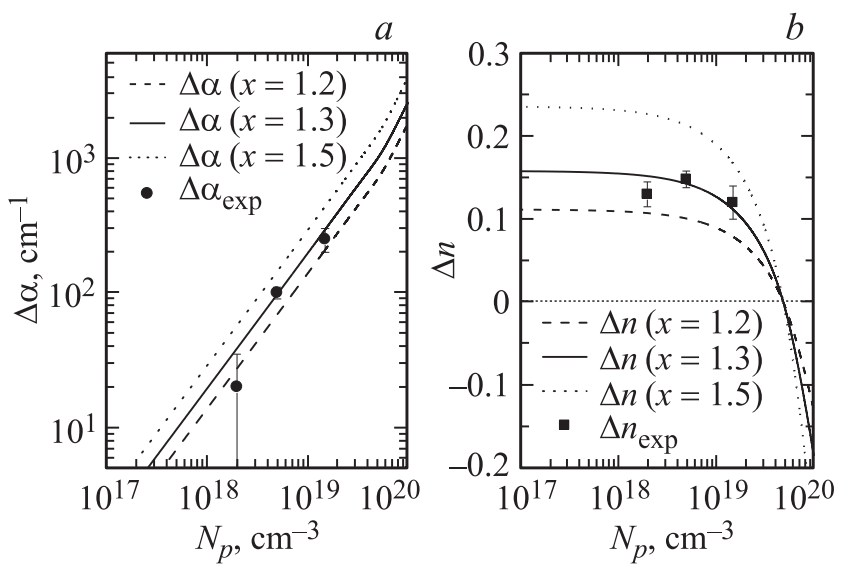

Pис. 3. $a-$ расчетные зависимости дихроизма $\Delta \alpha$ на частоте ИК излучения $1000 \mathrm{~cm}^{-1}$ при параметрах анизотропии $x=1.2$, 1.3 и 1.5 , величине затухания $\tau^{-1}=500 \mathrm{~cm}^{-1}$, от концентрации свободных носителей заряда в кремниевых нанокристаллах и данные эксперимента $\Delta \alpha_{\text {exp }}$ (точки) в зависимости от концентрации дырок в подложках, используемых при формировании анаизотропных слоев por-Si. $b-$ зависимости разности показателей преломления $\Delta n=n_{o}-n_{e}$ при тех же параметрах и экспериментальные значения $\Delta n_{\exp }$ (точки).

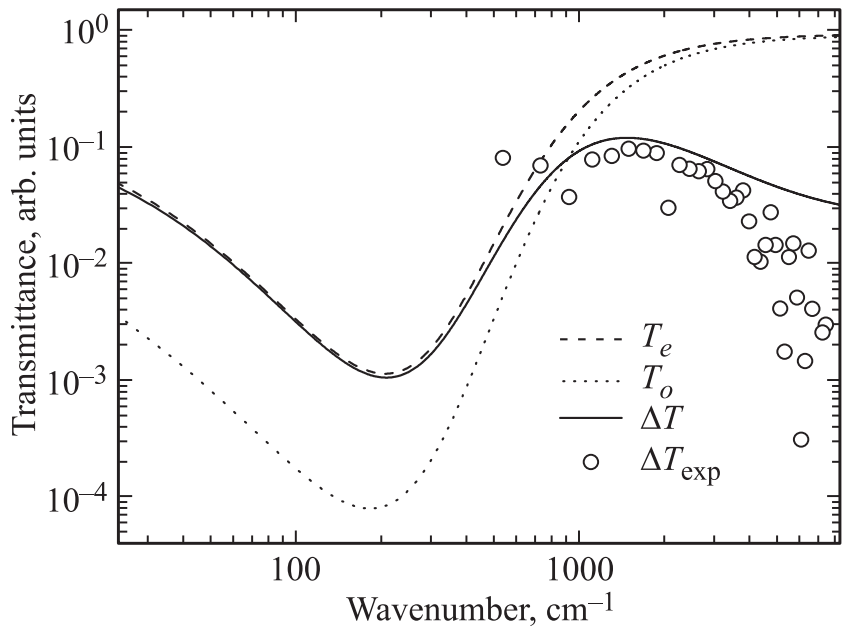

Рис. 4. Расчетные спектры пропускания $T_{e}$ (штриховая линия), $T_{o}$ (пунктирная линия), их разность $\Delta T$ (сплошная линия) для пленок por-Si (пористость $P=60 \%$, отношение полуосей $x=1.3$, величина затухания $\left.\tau^{-1}=500 \mathrm{~cm}^{-1}\right)$ и экспериментальные значения $\Delta T_{\exp }$ (кружки) из данных рис. 1.

различия экспериментальных и модельных спектров в более низкочастотной и высокочастотной областях спектра можно объяснить простотой используемой модели, не учитывающей взаимодействие света с колебаниями решетки и поверхностными молекулярными группами в нанокристаллах кремния, а также рассеяние света на структурных неоднородностях пленок por-Si. При рассматриваемой толщине пленки наблюдаемая немонотонная частотная зависимость $\Delta T$ обусловлена преимущественно дихроизмом. В то же время для меньших толщин, очевидно, также будет сказываться немонотонная спектральная зависимость коэффициента отражения, что может привести к более сложному спектру $\Delta T$.

Полученные результаты показывают, что оптические свойства слоев анизотропного пористого кремния могут сильно зависеть он концентрации подвижных носителей заряда. При этом вследствие дихроизма величина разностного пропускания достигает значений порядка $10 \%$. Рассмотренная анизотропия оптических свойств, очевидно, может быть связана не только с равновесными, но и с неравновесными, например, фотовозбужденными носителями заряда. В последнем случае анизотропные кремниевые слои могут быть использованы для модуляции интенсивности и фазы инфракрасного света в широком спектральном диапазоне спектра при помощи электрической инжекции или фотовозбуждения в области фундаментального поглощения.

\section{5. Заключение}

Таким образом, проведенные численное моделирование и экспериментальные исследования анизотропных оптических характеристик слоев por- $\mathrm{Si}$, полученных методом электрохимического травления сильно легирован- 
ных бором пластин $c$-Si, демонстрируют, что оптические свойства por-Si в ИК диапазоне спектра зависят от концентрации свободных носителей заряда. С увеличением концентрации носителей заряда происходит монотонный рост дихроизма и немонотонное изменение отражения и разностного пропускания слоев. Полученные результаты показывают, что слои анизотропного por-Si c контролируемой концентрацией подвижных носителей заряда могут быть использованы для управления интенсивностью прошедшего света в ИК области спектра. Обсуждаемые свойства важны для применения анизотропных слоев наноструктурированного кремния в устройствах фотоники и терагерцовой техники.

Работа была поддержана проектами Министерства образования и науки РК.

\section{Список литературы}

[1] O. Bisi, S. Ossicini, L. Pavesi. Surf. Sci. Rep., 38 (1), 1 (2000).

[2] Л.А. Головань, В.Ю. Тимошенко, П.К. Кашкаров. УФН, 177 (6), 619 (2007).

[3] V. Sivakov, S. Christiansen. J. Nanoelectr. Optoelectr., 7 (6), 583 (2012).

[4] N. Könzner, D. Kovalev, J. Diener, E. Gross, V.Yu. Timoshenko, G. Polisski, F. Koch, M. Fujii. Optics Lett., 26 (16), 1265 (2001).

[5] V.Yu. Timoshenko, L.A. Osminkina, A.I. Efimova, L.A. Golovan, P.K. Kashkarov, D. Kovalev, N. Künzner, E. Gross, J. Dienez, F. Koch. Phys. Rev. B, 67 (11), 113405 (2003).

[6] P.K. Kashkarov, L.A. Golovan, A.B. Fedotov, A.I. Efimova, L.P. Kuznetsova, V.Yu. Timoshenko, D.A. Sidorov-Biryukov, A.M. Zheltikov, J.W. Haus. J. Opt. Soc. Am. B, 19 (9), 2273 (2002).

[7] K.Q. Peng, Y.J. Yan, S.P. Gao, J. Zhu. Adv. Mater., 14 (16), 1164 (2002).

[8] L. Boarino, C. Boratto, F. Geobaldo, G. Amato, E. Comini, A.M. Rossi, G. Faglia, G. Lérondel, G. Sberveglieri. Mater. Sci. Eng. B, 69, 210 (2000).

[9] L. Pancheri, C.J. Oton, Z. Caburro, G. Soncini, L. Pavesi. Sensors Actuators B: Chem., 89 (3), 237 (2003).

[10] Л.А. Осминкина, Е.В. Курепина, А.В. Павликов, В.Ю. Тимошенко, П.К. Кашкаров. ФТП, 38 (5), 603 (2004).

[11] V.Yu. Timoshenko, L.A. Osminkina, A.I. Efimova, M.A. Fomenko, L.A. Golovan, P.K. Kashkarov, D. Kovalev, N. Künzner, E. Gross, J. Diener, F. Koch. Phys. Status. Solidi C, 2 (9), 3461 (2005).

Редактор Г.А. Оганесян

\section{Effect of free charge carriers on birefringence and dichroism in anisotropic porous silicon layers}

\author{
K.S. Sekerbayev ${ }^{\mathbf{1 , 2}}$, Y.T. Taurbayev ${ }^{\mathbf{1 , 2}}$, A.I. Efimova ${ }^{3}$, \\ V.Yu. Timoshenko ${ }^{3,4,9}$ T.I. Taurbayev ${ }^{1,2}$ \\ ${ }^{1}$ Institute Experimental and Theoretical Physics, \\ al-Farabi Kazakh National University, \\ 050040 Almaty, Kazakhstan \\ ${ }^{2}$ National Nanotechnology Laboratory of Open Type, \\ al-Farabi Kazakh National University, \\ 050040 Almaty, Kazakhstan \\ ${ }^{3}$ Lomonosov Moscow State University, \\ Department of Physics, \\ 119991 Moscow, Russia \\ ${ }^{4}$ National Research Nuclear University „MEPhl“, \\ 115409 Moscow, Russia
}

\begin{abstract}
Optical properties of anisotropic mesoporous silicon films containing free charge carriers (holes) were experimentally and theoretically studied in infrared spectral region. The simulation results of the optical properties of the prepared samples within the framework of the effective optical medium approximation show a strong dependence of the birefringence, anisotropy of the reflection and dichroism on the free charge carrier concentration. The nonmonotonic dependence of the differential transmittance spectra of the sample measured for the mutually perpendicular directions of light polarization is explained by considering a contribution of free charge carriers with concentration about $10^{19} \mathrm{~cm}^{-3}$. These results demonstrate that anisotropic silicon nanostructures are promising for applications in infrared photonics and terahertz devices.
\end{abstract}

